\title{
Ewing's Sarcoma/Pnet Presenting as Breast Mass
}

\author{
Pallavi Mishra*, Neha K Madan, Somshankar Chowdhury, Charanjeet Ahluwalia and Indrani Dhawan \\ Department of Pathology, VMMC and Safdarjung Hospital, India
}

Received: October 23, 2017; Published: October 31, 2017

*Corresponding author: Pallavi Mishra, Dept of Pathology, VMMC and Safdarjung Hospital, New Delhi, India, Tel: 7838618216;

Email: pallavi_1092@yahoo.co.in

\section{Abstract}

Introduction: Ewing's sarcoma family of tumours (ESFT) are uncommon, aggressive malignant tumours that usually arise in soft tissues or bones in children and young adults. Long bones of the lower limbs are most commonly involved. Occurrence at atypical sites can pose a diagnostic challenge. We report an exceptional case of this tumour presenting as a breast mass in a male patient.

Case Report: A 23-year-old male patient was referred to our centre with complaint of a progressively enlarging breast lump. This was initially suspected to be carcinoma, breast. On re-evaluation at our centre, FNAC yielded a diagnosis of small round cell malignancy and a possibility of Ewing's/PNET was suggested which was confirmed on trucut biopsy and immune histochemistry. Chest CT scan subsequently performed revealed a left sided heterogeneous soft tissue mass involving a large part of thorax, causing contra lateral meditational shift. Understanding the nature and extent of disease and expecting a dismal outcome the patient refused any further investigation and treatment.

Discussion: ESFT are aggressive fast growing tumours which require urgent diagnosis for swift commencement of therapy. Occurrence at unusual sites or uncommon presentations can lead to consideration of alternative clinical diagnoses and make the evaluation challenging. This case highlights the need for careful evaluation of cytology smears, keeping in mind the unexpected, avoiding the unnecessary diagnostic delay.

Abbreviations: ESFT: Ewing Sarcoma Family of Tumours; PNET: Peripheral Neuro Ectodermal Tumour; FNAC: Fine Needle Aspiration Cytology; CT: Computed Tomography

\section{Introduction}

Ewing Sarcoma Family of Tumours (ESFT) is uncommon, aggressive, malignant tumours that usually arise in soft tissue or bones in children and young adults. The term ESFT includes typical Ewing's Sarcoma, the Peripheral Neuro ectodermal tumour (PNET) or Askin'stumor of the chest wall [1]. It is the second most malignant tumour in the 10-20 year age group [2]. ESFTs arise from migrating embryonic cells of the neural crest showing variable neuro ectodermal differentiation. The differentiation pattern forms the basis of differentiating each individual type of ESFT though these features commonly overlap. This tumour family most commonly involves long bones of lower limbs, however, atypical presentations have been studied, which can present a diagnostic challenge $[3,4]$. Here we present an exceptional case of Ewing's Sarcoma presenting as a breast mass in a male patient.

\section{Case Report}

A 23 year old male presented with a four month history of a progressively enlarging lump in his left breast. Physical examination revealed a firm, fixed and non tender mass measuring approximately $8 \times 7$ centimetres in his left breast. It was unattached to the overlying skin. The contra lateral breast and both axilla were normal. A Fine Needle Aspiration Cytology (FNAC) performed at another tertiary centre concluded in the diagnosis of breast carcinoma and the patient was referred to our centre for further management. With a clinical suspicion of breast carcinoma in mind, a repeat FNAC was performed at our centre. Cytology smears showed loosely cohesive clusters of small to medium sized monomorphic round cells which were, at places arranged around blood vessels, against a background of necrotic debris. Cells had pale, vacuolated, fragile cytoplasm, round nuclei with opened up chromatin and 2-3 conspicuous nucleoli (Figures 1a \& 1b).

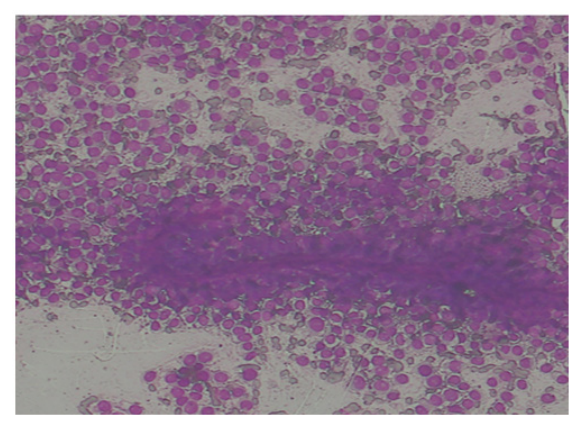

Figure 1a: Loosely cohesive clusters of small to medium sized monomorphic round cells against a necrotic background. 


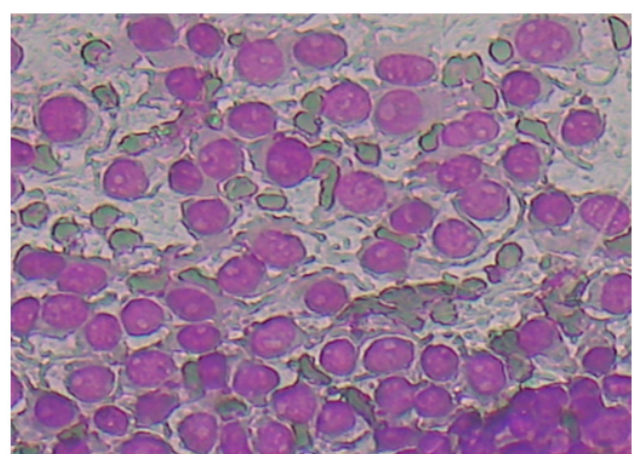

Figure 1b: These cells have pale, vacuolated, round nuclei with opened up chromatin and 2-3 conspicuous nucleoli.

Based on these features a diagnosis of small round cell malignancy with a possibility of Ewing's Sarcoma/PNET was suspected. Trucut biopsy was performed and showed small round tumour cells arranged around blood vessels with occasional rosette formation and areas of necrosis (Figure 2a). On immune histochemistry cells stained positive for CD 99, NSE and vimentin (Figures 2b-2d) while they were negative for LCA, synaptophysin, cytokeratin and desmin. This confirmed the diagnosis of Ewing's Sarcoma/ PNET. Computed tomography (CT) scan showed a large, heterogeneously enhancing, solid left chest wall mass with internal necrosis arising from anterior fourth rib, with extension into left breast, mediastinal shift and complete atelectasis of the left lung (Figure 3).

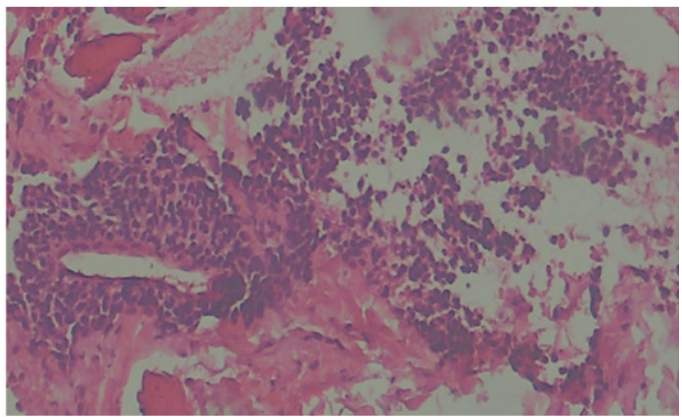

Figure 2a: Small round tumor cells arranged around blood vessels with occasional rosette formation and areas of necrosis.

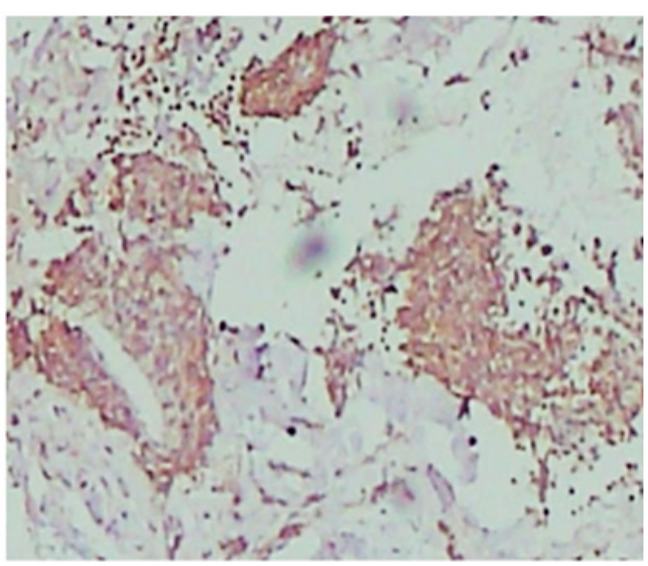

Figure 2b: Positivity for CD99.

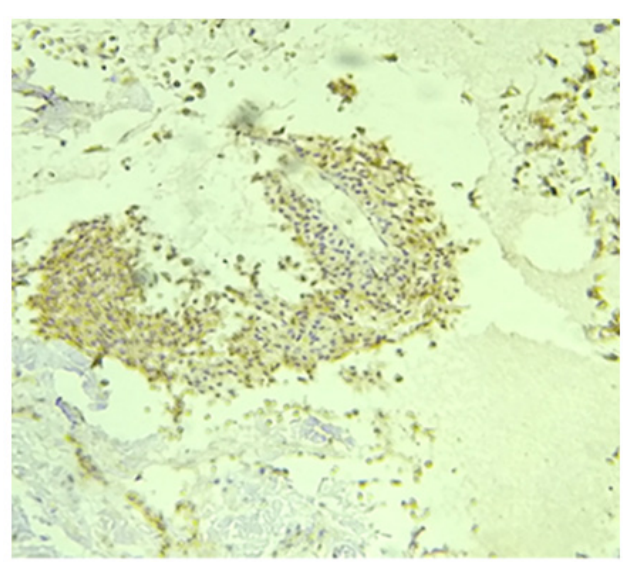

Figure 2c: Positivity for vimentin.

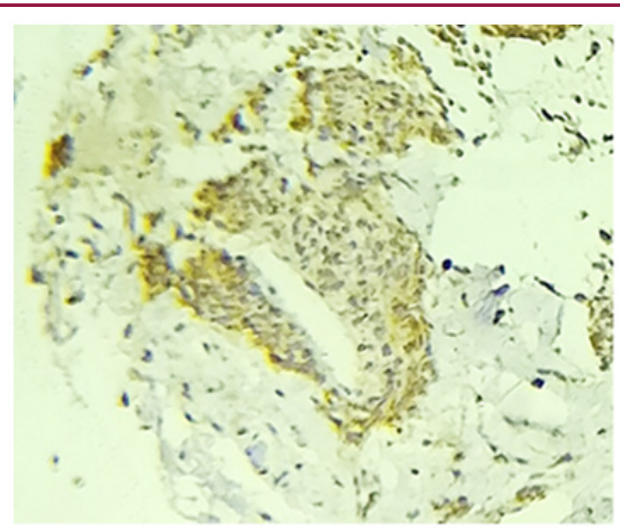

Figure 2d: Positivity for NSE.

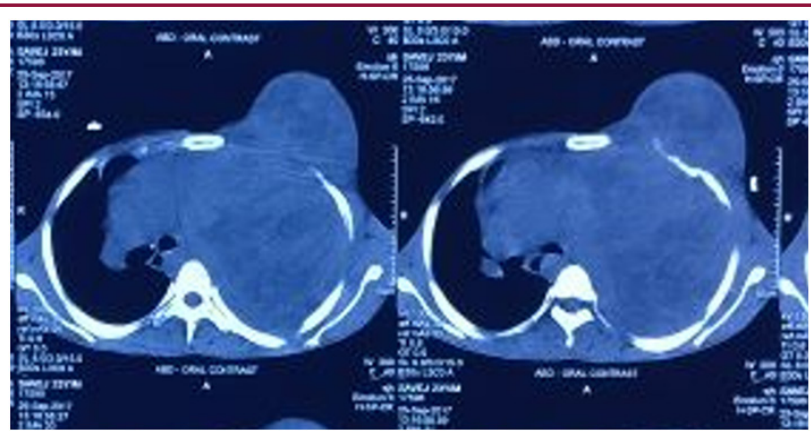

Figure 3: Computed tomography $(\mathrm{CT})$ Scan showed a large, heterogeneously enhancing, solid left chest wall mass with internal necrosis arising from anterior fourth rib, with extension into left breast, mediastinal shift and complete atelectasis of the left lung.

\section{Discussion}

ESFTs form a single group of bone and soft tissue tumours with undifferentiated Ewing's at one end of the spectrum and Peripheral neuro ectodermal tumour (PNET) showing clear signs of neural differentiation at the other [5]. The most common sites of Ewing's sarcoma are chest wall, Para vertebral region, retroperitoneal space, lower extremities, and gluteal region. However, few cases have been reported in the kidney, breast, gastrointestinal tract, prostate, endometrium, the adrenal glands, brain, and lung [6]. The breast is uncommonly involved and only 5-6 cases have been reported in 
English literature, all of them in females [7]. This case is probably the first case of this tumour presenting as a breast mass in a male patient. Occurrence at unusual sites or uncommon presentations can lead to consideration of alternative clinical diagnoses and make the evaluation challenging. The index case had a tumorprobably arising from a rib, extending into thorax posteriorly and presenting as a breast mass anteriorly. This resulted in an initial confounding diagnosis of carcinoma breast.

A delay in the correct diagnosis resulted in expansion of the tumour into the mediastinum, thus upstaging the disease. It has been reported that those with localized non pelvic disease treated with multimodal therapeutic modalities including concomitant chemoradiation, have a5-year disease-free survival rate of approximately $70 \%$. This, however, comes down significantly when the tumour spreads into the body cavities, as was seen in this case. The patient belonged to a low socio economic background and understanding the poor prognosis, he refused any further investigations and treatment. On FNAC, the differential diagnoses consists of other small round cell tumours like lymphoma, plasmacytoma, as well as small cell variants of the various epithelial and soft tissue tumours. Careful evaluation of the cytology smears, keeping in mind the unexpected, and supplementing it with immune histochemical marker studies, is, therefore, of utmost importance.

This group of tumours is characterized by the presence of the typical translocation $\mathrm{t}(11 ; 22)$ (q24; q12), the EWS-FLI1 chimeric transcript on molecular analysis and the expression of CD99 antigen (MIC-2) at immune histochemistry [8]. Other markers, like vimentin and NSE, show variable positivity. Imaging modalities usually present findings that are non specific. The diagnosis is usually made on histopathology, immunohistochemistry and the characteristic translocation $t(11 ; 22)$ found on molecular analysis. This case, however, highlights the importance of thorough evaluation of FNAC smears in guiding towards diagnosis and also the need for an early diagnosis in such cases.

\section{Conclusion}

ESFTs are aggressive, fast growing tumours which require urgent diagnosis for swift commencement of therapy. Occurrence at unusual sites or uncommon clinical presentations can lead to consideration of alternate clinical diagnosis, making the management challenging. The delay in diagnosis in the index case might have led to the spread of disease and a dismal prognosis, which should be avoided.

\section{References}

1. Machado I, Noguera R, Mateos EA Calabuig-Fariñas S, López FI, Martínez A, et al. (2011) the many faces of atypical Ewing's sarcoma. A true entity mimicking sarcomas, carcinomas and lymphomas. Virchows Arch 458(3): 281-290.

2. Sugeeth MT, Azariah B, PreethiT, Nair SG (2016) an unusual breast tumor-A case report. J Med Case Rep 4(10): 775-776.

3. Basma EE, Hajar H, Nabil M, Anis B, Samir B (2012) Breast Ewing sarcoma/primitive neuroectodermaltumor: A case report and a review of the literature. Breast 3(10): 5.

4. Shin JH, Lee HK, Rhim SC, Cho KJ, Choi CG, et al. (2001) Spinal epidural extra skeletal Ewing sarcoma: MR findings in two cases. AJNR Am J Neuroradiol 22(4): 795-798.

5. Dehner LP (1993) Primitive neuro ectodermaltumor and Ewing's sarcoma. Am J Surg Pathol 17(1): 1-13.

6. Basma EE, Hajar H, Nabil M, Anis B, Samir B (2012) Breast Ewing sarcoma/primitive neuroectodermaltumor: A case report and a review of the literature. Breast 3(10): 5 .

7. An Unusual Tumor of the Breast-Extraskeletal Ewing Sarcoma.

8. Folpe AL, Goldblum JR, Rubin BP, Shehata BM, Liu W, et al. (2005) Morphologic and immune phenotypic diversity in Ewing family tumors: a study of 66 genetically confirmed cases. Am J Surg Pathol 29: 10251033.

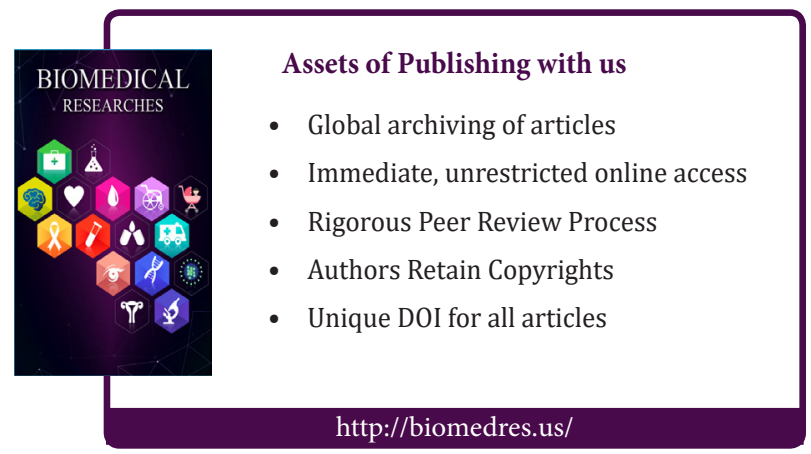

